\title{
Hepatitis B virus X protein sensitizes HL-7702 cells to oxidative stress-induced apoptosis through modulation of the mitochondrial permeability transition pore
}

\author{
WEN-YU GAO ${ }^{1 *}$, DAN LI ${ }^{1 *}$, DE-EN CAI ${ }^{2}$, XIAO-YUN HUANG ${ }^{2}$, BI-YUN ZHENG $^{1}$, \\ YUE-HONG HUANG ${ }^{1}$, ZHI-XIN CHEN ${ }^{1}$ and XIAO-ZHONG WANG ${ }^{1}$ \\ ${ }^{1}$ Department of Gastroenterology, Fujian Medical University Union Hospital, Fujian 350001; \\ ${ }^{2}$ Graduate School, Fujian Medical University, Fujian 350001, P.R. China
}

Received April 30, 2016; Accepted June 10, 2016

DOI: 10.3892/or.2016.5225

\begin{abstract}
Chronic hepatitis B virus (HBV) infection is a leading cause of liver cirrhosis and cancer. Among the pathogenic factors of $\mathrm{HBV}, \mathrm{HBV} \mathrm{X}$ protein $(\mathrm{HBx})$ is attracting increased attention. Although it is documented that $\mathrm{HBx}$ is a multifunctional regulator that modulates cell inflammation and apoptosis, the exact mechanism remains controversial. In the present study, we explored the effect of HBx on oxidative stress-induced apoptosis in normal liver cell line, HL-7702. Our results showed that the existence of HBx affected mitochondrial biogenesis by modulating the opening of the mitochondrial permeability transition pore (MPTP). Notably, this phenomenon was associated with a pronounced translocation of Bax from the cytosol to the mitochondria during the period of exposure to oxidative stress with a release of cytochrome $c$ and activation of cleaved caspase-3 and PARP. Moreover, MPTP blockage with cyclosporin A prevented the translocation of Bax, and inhibited oxidative stress-induced apoptotic killing in the HBx-expressing HL-7702 cells. Our findings suggest that HBx exhibits pro-apoptotic effects upon normal liver cells following exposure to oxidative stress by modulating the MPTP gateway.
\end{abstract}

Correspondence to: Professor Xiao-Zhong Wang, Department of Gastroenterology, Union Hospital of Fujian Medical University. 29 Xinquan Road, Gulou, Fuzhou, Fujian 350001, P.R. China E-mail: drwangxz@163.com

${ }^{*}$ Contributed equally

Abbreviations: Bcl-2, B-cell lymphoma-2; Bax, Bcl-2-associated X protein; COX, cytochrome $c$ oxidase; CsA, cyclosporin $\mathrm{A} ; \mathrm{H}_{2} \mathrm{O}_{2}$, hydrogen peroxide; $\mathrm{HBV}$, hepatitis $\mathrm{B}$ virus $\mathrm{X}$ protein; $\mathrm{HCC}$, hepatocellular carcinoma; DCFH-DA, 2',7'-dichlorofluorescein diacetate; HBx, hepatitis B virus X protein; MPTP, mitochondrial permeability transition pore; PBS, phosphate-buffered saline; ROS, reactive oxygen species; VDAC, voltage-dependent anion channel

Key words: hepatitis B virus $\mathrm{X}$ protein, mitochondrial permeability transition pore, oxidative stress, Bax, apoptosis

\section{Introduction}

Hepatitis B virus (HBV) infection results in a significantly high risk of severe liver diseases such as hepatic cirrhosis and hepatocellular carcinoma (HCC). The hepatitis B virus X protein (HBx), which is a $17-\mathrm{kDa}$ non-structural protein consisting of 154 amino acids, is considered as a key regulatory viral protein in HBV replication and virus-associated liver diseases (1).

HBx is a multifunctional protein that regulates numerous cellular signal transduction pathways and participates in cell proliferation, differentiation, cell cycle progression, autophagy and apoptosis $(2,3)$. The specific function of HBx is linked to its subcellular localization (4). It is generally believed that $\mathrm{HBx}$ is mostly cytoplasmic, with a small fraction in the nucleus and many groups have reported that the mitochondrion is a major target for HBx in the cytoplasm $(5,6)$, causing mitochondrial damage by regulating mitochondrial membrane potential (7), increasing the generation of cellular reactive oxygen species (ROS) $(8,9)$ and modulating the opening of the mitochondrial permeability transition pore (MPTP). Studies in HepG2 cells and primary rat hepatocytes suggest that MPTP activity is required for HBV replication and the expression of cell cycle proteins modulated by HBx $(10,11)$. Previous studies have observed the release of cytochrome $c$ from purified mitochondria after the induction of MPT and therefore induced apoptosis (12), identifying MPTP as an intracellular sensor of oxidants and other toxins. However, whether HBx-induced apoptosis is related to its function of modulating MPTP and the specific mechanism have not been fully understood.

Since apoptosis has been implicated as a vital mechanism for inflammation and hepatocarcinogenesis (13), a large body of research has tried to explore the role of HBx in cell apoptosis and its contribution to HBV-associated HCC. The results of these studies are controversial; HBx has been shown to induce $(14,15)$, inhibit $(16,17)$ or have no effect on apoptosis (18). The discrepancy of the role of HBx on apoptosis may be due to the different cell types, culture condition or experimental systems used in different studies. In addition, some of these studies have demonstrated that HBx did not induce cell apoptosis itself, but instead sensitized hepatocytes to a variety of apoptotic signals such as TNF- $\alpha$, TRAIL, ethanol, Fas and 
oxidative stress $(19,20)$. Notably, oxidative stress has been implicated in DNA damage and apoptosis, contributing to the pathogenesis of inflammatory diseases and cancer $(13,21)$.

The Bcl-2 protein family plays a pivotal role in $\mathrm{HBx}$-induced cell apoptosis. They are divided into anti-apoptotic members (Bcl-2, Bcl-xL and Mcl-1) and pro-apoptotic members (Bax, Bak and Bid) (22). Bax is one of the pro-apoptotic members of the Bcl-2 protein family and is considered as a key factor of the intrinsic apoptosis pathway upon various stimuli. In non-apoptotic cells, it is in dynamic equilibrium between the mitochondrion and cytosol. In response to apoptotic stimulus, Bax changes its conformation, disrupting the equilibrium and causing Bax to accumulate at the mitochondrion (23). A previous study in serum-starved HepG2 cells demonstrated that HBx induced the translocation of Bax to the mitochondrion (24), suggesting that Bax functions as an important element in the $\mathrm{HBx}$-induced intrinsic apoptosis pathway. Other studies also demonstrated that Bax plays an important role in $\mathrm{H}_{2} \mathrm{O}_{2}$-induced apoptosis via its mitochondrial translocation (25). Recent studies have confirmed that the translocation of Bax in apoptosis is dependent on voltage-dependent anion channel (VDAC) 2 (26), which is thought to be a component of MPTP (27).

In the present study, we reported a possible function for HBx to modulate MPTP in the hepatic HL-7702 cell line, and confirmed that this function was associated with oxidative stress-induced apoptosis through translocation of Bax. These new findings may have implications for understanding the role of HBx in HBV-associated inflammation and hepatocarcinogenesis.

\section{Materials and methods}

Cell culture and transfection. The human HL-7702 hepatocyte cell line was purchased from the Shanghai Cell Bank (Shanghai, China). The cells were grown in Dulbecco's modified Eagle's medium (DMEM) containing $10 \%$ fetal bovine serum (FBS) and maintained at $37^{\circ} \mathrm{C}$ in a humidified atmosphere composed of $95 \%$ air and $5 \% \mathrm{CO}_{2}$. Cultures of HL-7702 cells were transfected with recombinant plasmids using the transfection reagent Lipofectamine 3000 (Invitrogen, Carlsbad, CA, USA) according to the manufacturer's protocol.

Plasmids. The recombinant plasmid pGEM-HBV, which expresses a greater-than-genome-length cDNA of wild-type HBV (ayw) and pGEM-HBV-HBx, which is identical to HBx-deficient mutant $\mathrm{HBV}$, were a gift from Professor M.J. Bouchard (Drexel University, Philadelphia, PA USA) $(28,29)$. The HBx expression plasmid pcDNA3.1-X was kept in our laboratory. We designed new pcDNA3.1 plasmids expressing HBx fused to the eight amino acid FLAG epitope using oligonucleotides containing terminal XbaI or EcoRI restriction enzyme sites by PCR. The forward oligonucleotide sequence for HBx-flag was: 5'-GCT^CTAGAGCCACCATGG CTGCTAGGCTGTGCT-3' and the reverse oligonucleotide sequence was, 5'-GCCTTAA^GTTACTTATCATCGTCGTC CTTGTAGTCGGCAGAGGTGAAAAAG-3'. XbaI or EcoRI enzymes (New England Biolabs, Ipswich, MA, USA) were used for the digestion of insert and vector, and digestion prod- ucts were followed by ligation with T4 DNA ligase (Promega, Madison, WI, USA).

Western blot analysis. Cells were lysed in RIPA buffer (Beyotime, Shanghai, China) supplemented with protease inhibitors in ice for $30 \mathrm{~min}$ followed by centrifugation at $12,000 \mathrm{x} \mathrm{g}$ for $15 \mathrm{~min}$ at $4^{\circ} \mathrm{C}$. Immunoblotting was performed using the anti-cleaved PARP mAb (1:1,000), anti-Bax mAb (1:1,000; Cell Signaling Technology, Danvers, MA, USA), anti-cytochrome $c$ mAb (1:500), anti-caspase-3 mAb (1:1,000; Abcam, Cambridge, MA, USA), anti-HBc mAb (1:500; Milipore, Billerica, MA, USA), anti- $\beta$-actin and anti-secondary antibodies were purchased from ZSGB-BIO (Beijing, China).

Isolation of mitochondria and measurement of COX activity. Cells transfected with recombinant plasmids were lysed and the mitochondria were isolated using the mitochondrion isolation kit for mammalian cells (Thermo Fisher Scientific, Inc., Waltham, MA, USA). Protein concentrations were measured using the bicinchoninic acid (BCA) assay (Beyotime) and COX activity was determined using the Cytochrome $c$ Oxidase Assay kit (GenMed, Shanghai, China) as previously described $(8,9)$.

ATP measurement. Cell viability of the transfected cells was assessed using a luciferase-coupled ATP quantitation assay (Promega). Transfected cells were dispensed in culture medium at $20,000 \mathrm{cells} / 100 \mu \mathrm{l} /$ well into 96 -well white/solid-bottom assay plates. The assay plates were equilibrated at room temperature for $30 \mathrm{~min}$, followed by the addition of $100 \mu \mathrm{l} /$ well of CellTiter-Glo reagent and the contents were mixed for $2 \mathrm{~min}$ on an orbital shaker to induce cell lysis. The assay plates were allowed to incubate at room temperature for $10 \mathrm{~min}$ to stabilize the luminescent signal. Then, the luminescence intensity of the plates was assessed using a luminometer (Orion II Microplate Luminometer; Berthold Detection Systems GmbH, Pforzheim, Germany).

Mitochondrial membrane potential analysis. The cultured HL-7702 cells were seeded and transfected in 6-well plate for $36 \mathrm{~h}$, and the mitochondrial membrane potential was evaluated using the cationic fluorescent dye Rhodamine-123 (Sigma, St. Louis, MO, USA). Transfected cells were digested and washed with phosphate-buffered saline (PBS). Diluted Rhodamine-123 (10 mg Rhodamine-123/ml with serum-free DMEM) was added to the cultures and incubated at $37^{\circ} \mathrm{C}$ for $30 \mathrm{~min}$. The cells were washed and resuspended in PBS for analysis by flow cytometry (BD Biosciences, San Jose, CA, USA).

Cytosolic calcium imaging assay. Cultured HL-7702 cells were seeded and transfected in a 24-well plate for $36 \mathrm{~h}$ with the addition of $1 \mu \mathrm{M}$ cyclosporin $\mathrm{A}$ (CsA) (Milipore) or dimethyl sulfoxide (DMSO) for $12 \mathrm{~h}$. Levels of cytosolic calcium were measured by Flou-4 calcium imaging kit (Molecular Probes, Eugene, OR, USA) following the manufacturer's instructions. Fluorescence images were obtained (excitation $494 \mathrm{~nm} /$ emission $506 \mathrm{~nm}$ ) by confocal laser scanning microscopy (Leica SP5; Leica, Solms, Germany). Relative fluorescence intensity of each group was measured by LAS AF Lite software (Leica). 
A

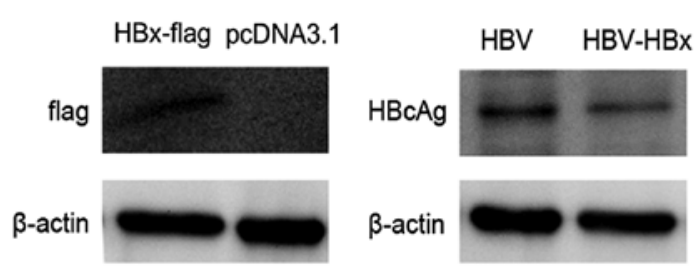

C

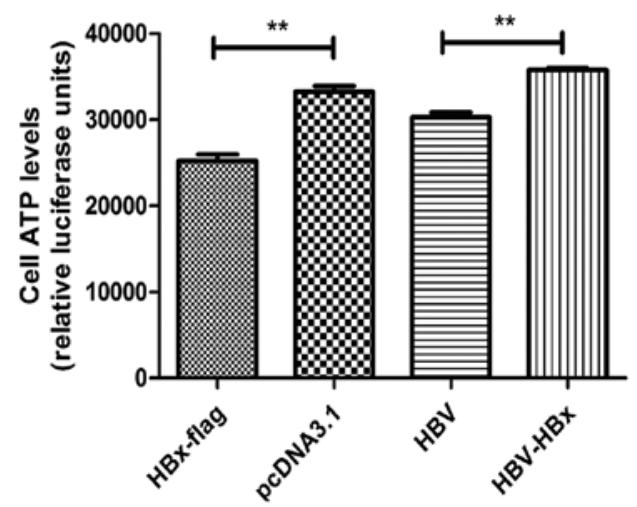

B
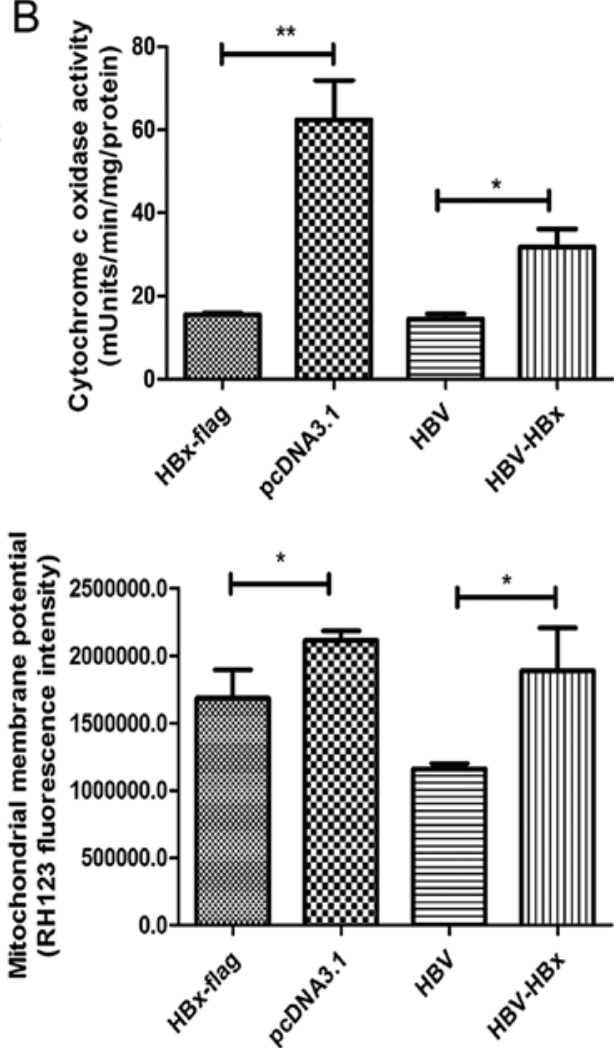

Figure $1 . \mathrm{HBx}$ reduces COX activity to modulate mitochondrial membrane potential and cell viability. (A) $\mathrm{HBx}$-flag fusion proteins and $\mathrm{HBcAg}$ proteins were transiently expressed in HL-7702 cells. (B) Mitochondria were isolated form transfected cells and changes in COX activity were assessed by COX activity assay kit. (C) Cell ATP levels were examined by CellTiter-Glo Luminescent cell viability assay. (D) Mitochondrial membrane potential was measured by fluorescence dye, $\mathrm{RH} 123$, followed by flow cytometric analysis. Values shown represent the mean $\pm \mathrm{SD}$ of three independent experiments; ${ }^{*} \mathrm{P}<0.05$ and ${ }^{* *} \mathrm{P}<0.01$.

Fluorescence assay of ROS. Assay of intracellular ROS was preformed using 2',7'-dichlorofluorescein diacetate (DCFH-DA; Sigma). Cultured HL-7702 cells were plated and transfected into 24-well plate for $36 \mathrm{~h}$ with or without the following treatment of hydrogen peroxide $\left(\mathrm{H}_{2} \mathrm{O}_{2}\right)$ at the final concentration of $250 \mu \mathrm{M}$ (Sigma). Culture medium was first removed, and the cells were washed three times with serum-free DMEM, and diluted DCFH-DA $(10 \mu \mathrm{M}$ DCFH-DA with serum-free DMEM) was added to the cultures and incubated at $37^{\circ} \mathrm{C}$ for $30 \mathrm{~min}$ at the same end time point. The fluorescence was measured at $485 \mathrm{~nm}$ for excitation and $530 \mathrm{~nm}$ for emission within $60 \mathrm{~min}$ by confocal laser scanning microscopy (Leica SP5). Relative fluorescence intensity of each group was measured by LAS AF Lite (Leica).

Indirect immunofluorescence assay. Transfected cells were exposed to the indicated amount of $\mathrm{H}_{2} \mathrm{O}_{2}(250 \mu \mathrm{M})$ for $12 \mathrm{~h}$ with or without pretreatment with $1 \mu \mathrm{M} \mathrm{CsA}$ or DMSO for $30 \mathrm{~min}$. Cells were transplanted in 24-well plate cell slides and stained for 30 min with $150 \mathrm{nM}$ MitoTracker Red (Molecular Probes). After treatment, the cells were fixed with $4 \%$ ice paraformaldehyde for $30 \mathrm{~min}$, permeabilized with $3 \%$ Triton X-100 (PBST) for $10 \mathrm{~min}$ and incubated in blocking solution (5\% donkey serum albumin in PBST) for $45 \mathrm{~min}$. Then, the cells were incubated with primary antibodies (anti-Bax; 1:100) at $4^{\circ} \mathrm{C}$ overnight. The fluorescence-labeled secondary antibodies (donkey anti-rabbit fluorescence-labeled secondary antibodies;
Alexa Fluor ${ }^{\circledR}$ 488-conjugated; 1:500; Molecular Probes) were added and incubated for $60 \mathrm{~min}$ at $37^{\circ} \mathrm{C}$ in the dark, and the sections were mounted using Antifade mounting medium (Beyotime). Fluorescence images were obtained by confocal laser scanning microscopy (Leica SP5).

Apoptosis assay. Transfected cells were exposed to the indicated amount of $\mathrm{H}_{2} \mathrm{O}_{2}$ at the final concentration of $250 \mu \mathrm{M}$ for $12 \mathrm{~h}$ with or without pretreatment with $1 \mu \mathrm{M}$ CsA or DMSO for $30 \mathrm{~min}$. The extent of apoptosis was evaluated using the Annexin V-FITC apoptosis detection kit (BD Biosciences) by flow cytometric analysis.

Statistical analysis. Each set of experiments was repeated at least three times with similar results. The values given are presented as mean \pm SD. Statistical analysis was performed using the Student's t-test. In all cases, $\mathrm{P}<0.05$ was considered to indicate a statistically significant result.

\section{Results}

Intracellular expression of recombinant plasmids in transfected cells. Cultures of HL-7702 cells transiently transfected with recombinant pcDNA3.1, HBx-flag, pHBV, pHBV-HBx and $\mathrm{HBx}$-flag fusion protein and $\mathrm{HBcAg}$ were detected by western blotting for assessment of $\mathrm{HBx}$-flag and $\mathrm{pHBV} / \mathrm{pHBV}$ HBx (Fig. 1A). 

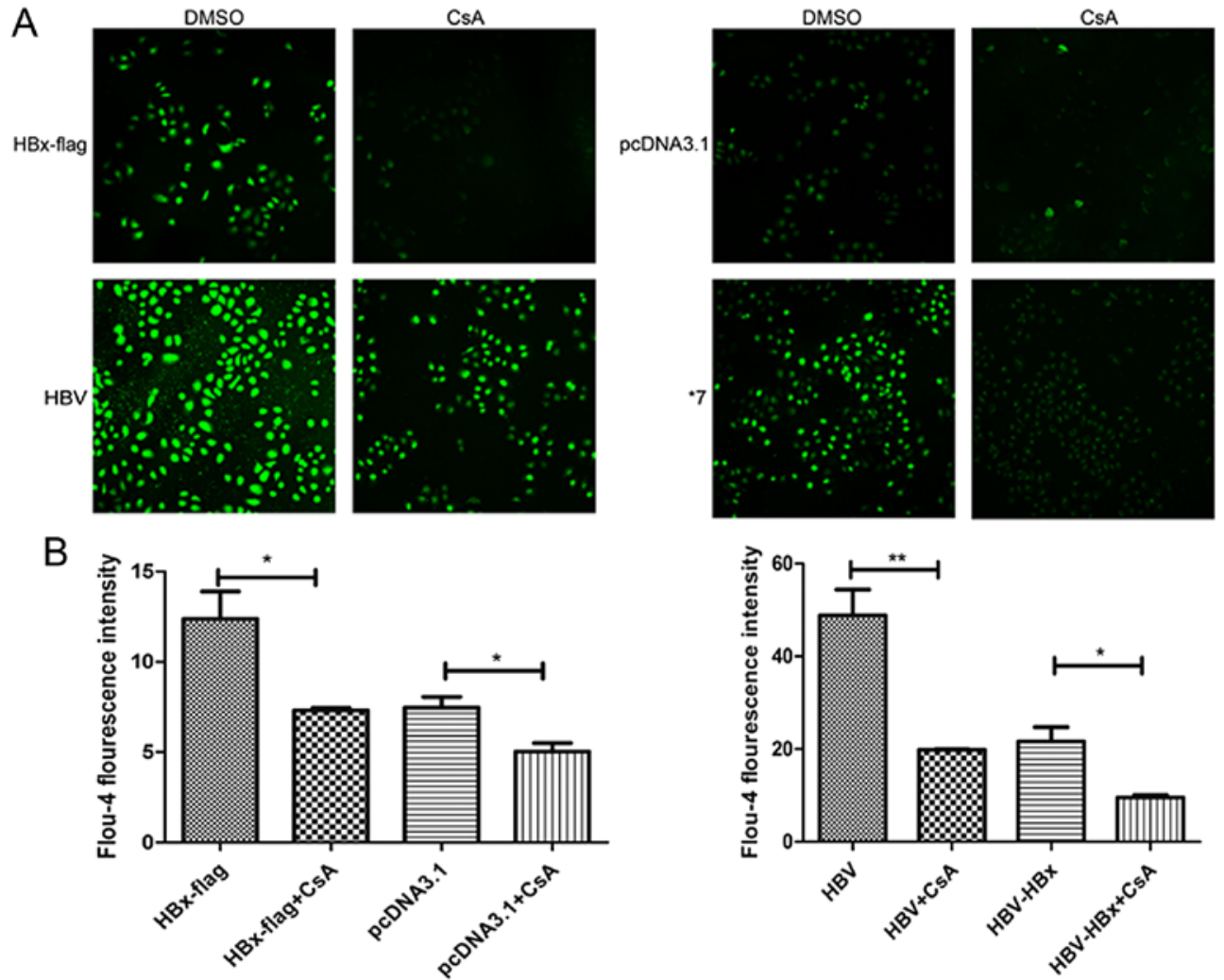

Figure 2. HBx increases the cytosolic calcium levels by modulating MPTP in HL-7702 cells. (A) Transfected cells treated with or without CsA were loaded with Flou-4 dye for $30 \mathrm{~min}$. Fluorescence images were obtained by confocal laser scanning microscopy. Magnification, x1,000. (B) Relative fluorescence intensity of each group was recorded. Values shown represent the mean $\pm \mathrm{SD}$ of three independent experiments; ${ }^{*} \mathrm{P}<0.05$ and ${ }^{* *} \mathrm{P}<0.01$.
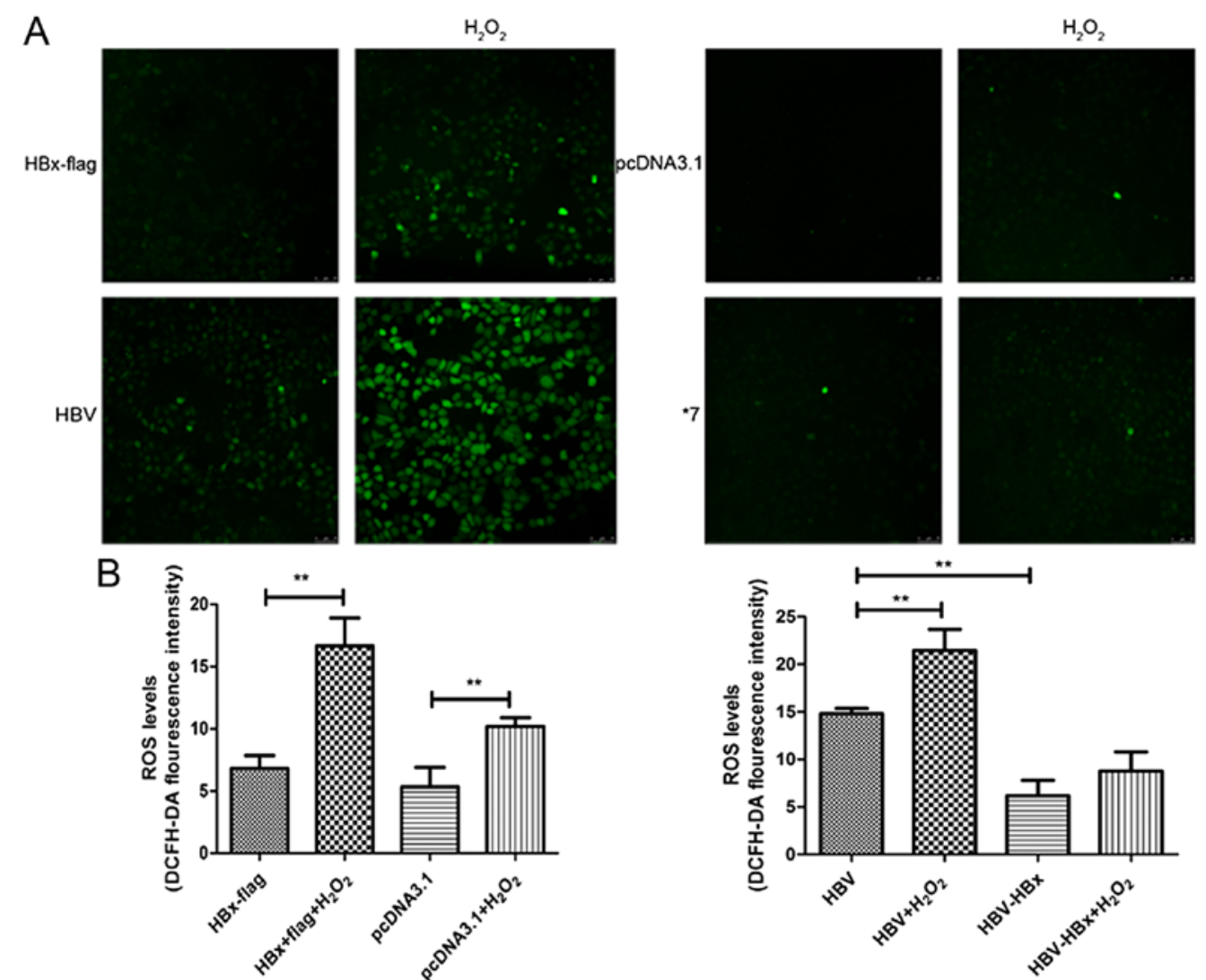

Figure 3. HBx increases intracellular ROS and sensitizes HL-7702 cells to oxidative stress. (A) Transfected cells treated with or without $\mathrm{H}_{2} \mathrm{O}_{2}$ were loaded with DCFH-DA dye for $30 \mathrm{~min}$. Fluorescence images were captured by confocal laser scanning microscopy. Magnification, x1,000. (B) Relative fluorescence intensity of each group was recorded. Values shown represent the mean $\pm \mathrm{SD}$ of three independent experiments; ${ }^{* *} \mathrm{P}<0.01$. 
HBX reduces COX activity to decrease cell ATP levels and modulate mitochondrial membrane potential. $\mathrm{COX}$ is a vital component of the mitochondrial respiratory chain. After transfections, a significant decrease in COX activity was noted in the HBx-flag- and pHBV-transfected cells compared with the activity in the cells transfected with pcDNA3.1 and pHBVHBx (Fig. 1B). Correlated with the downregulation of COX activity, we found an obvious decrease in cell ATP levels in the HBx-flag- and pHBV-transfected cells vs. that in the cells transfected with pcDNA3.1 and pHBV-HBx (Fig. 1C). Consistently, flow cytometric analysis of Rhodamine-123-stained cells was used to determine the effects of $\mathrm{HBx}$ on mitochondrial membrane potential (Fig. 1D). Results for the four groups indicated that $\mathrm{HBx}$ reduced the mitochondrial membrane potential in the HL-7702 cells. All the results suggest that since COX is essential for the generation of cell ATP and maintenance of mitochondrial membrane potential (30), the reduction in $\mathrm{COX}$ activity by $\mathrm{HBx}$ may be the initial factor of $\mathrm{HBx}$-induced mitochondrial dysfunction, including regulation of MPTP and induction of the mitochondrial apoptosis pathway.

HBX increases the cytosolic calcium levels via modulating MPTP in HL-7702 cells. The transfected cells were loaded with $\mathrm{Ca}^{2+}$ sensitive dye Fluo-4 to monitor the occurrence of cytosolic calcium levels. As shown in Fig. 2A and B, Fluo-4 fluorescence was significantly increased in the $\mathrm{HBx}-$ and pHBV-expressing cells vs. that in the control group. The result was consistent with a previous study in HepG2 cells (11). To determine whether the association with mitochondria is involved in the HBx-induced elevation of cytosolic calcium, we next examined the cytosolic calcium levels in the four groups treated with CsA $(1 \mu \mathrm{M})$, a specific inhibitor of MPTP. The data showed that $1 \mu \mathrm{M} \mathrm{CsA}$ partially decreased the cytosolic calcium concentrations in four groups. Meanwhile, following treatment with CsA, cytosolic calcium of HBx-flag cells was reduced to a level similar to pcDNA3.1 cells without CsA. Similarly, pHBV cells also exhibited a reduction in fluorescence intensity with CsA to the extent of pHBV-HBx cells, which indicated that $1 \mu \mathrm{M}$ CsA significantly blocked the function of HBx in modulating MPTP.

HBx increases intracellular ROS and sensitizes HL-7702 cells to oxidative stress. To further evaluate whether susceptibility of HL-7702 cells under oxidative stress conditions could be disturbed by $\mathrm{HBx}$, cells with or without $\mathrm{H}_{2} \mathrm{O}_{2}$ treatment were incubated with DCFH-DA and fluorescence was evaluated by confocal laser scanning microscopy (Fig. 3A). Notably, no apparent change was noted in the $\mathrm{HBx}$-expressing cells compared with the control group without treatment of $\mathrm{H}_{2} \mathrm{O}_{2}$, while a significant increase in fluorescence intensity was observed in the pHBV-expressing cells compared with that in the pHBV-HBx-expressing cells. However, although increased ROS levels were observed in all four groups when exposed to $\mathrm{H}_{2} \mathrm{O}_{2}$, an increased number of DCFH-DA-positive cells was found in the $\mathrm{H}_{2} \mathrm{O}_{2}$-exposed $\mathrm{HBx}$-expressing cells and pHBV-expressing cells than the control groups under the same condition (Fig. 3B).

$H B x$ induces translocation of Bax to mitochondria upon exposure to $\mathrm{H}_{2} \mathrm{O}_{2}$ via modulating MPTP. Bax is the key effector of the intrinsic apoptotic pathway initiated in response to diverse stimuli $(23,31)$. Evidence indicates that Bax maintains a balance between the cytosol and mitochondria in the non-apoptotic state, while under the stimulation of apoptosis, cytosolic Bax changes its formation and then insert into the mitochondrial outer membrane, forming large channels at the mitochondrial outer membrane leading to the release of cytochrome $c$ (32). Therefore, translocation of Bax from the cytosol to the mitochondrion may be an essential step in mitochondrial-mediated apoptosis. HBx has been shown to induce the translocation of Bax under serum starvation condition in $\mathrm{HepG} 2$ cells (24). To explore the state of Bax in $\mathrm{HBx}$-expressing cells upon $\mathrm{H}_{2} \mathrm{O}_{2}$ in normal hepatocytes, we proceeded with fluorescent staining experiments by a combination of anti-Bax antibody and MitoTracker Red. As shown in Fig. 4A, the staining of Bax (green color) along with mitochondria (red color) was observed (yellow) in the $\mathrm{HBx}$-flag cells exposed to $\mathrm{H}_{2} \mathrm{O}_{2}$ while the $\mathrm{HBx}$-flag cells without treatment of $\mathrm{H}_{2} \mathrm{O}_{2}$ did not show a visible co-localization of Bax and mitochondria, and this translocation of Bax was blocked by pretreatment of CsA. In addition, western blot analysis revealed that the whole protein levels of Bax did not show an apparent change in each group. While the expression of Bax protein exhibited a significant decrease in the cytosol and an increase in the mitochondria in the HBx-expressing cells treated with $\mathrm{H}_{2} \mathrm{O}_{2}$, pretreatment with CsA blocked the translocation of Bax (Fig. 4B). Both results suggest that $\mathrm{HBx}$ induced translocation of Bax to the mitochondria during oxidative stress, and MPTP blockage with CsA recovered the balance of Bax between the mitochondria and the cytosol in the HL-7702 cells.

MPTP blockage with CsA attenuates the pro-apoptotic effect of HBx in response to oxidative stress. It has been reported that $\mathrm{HBx}$ sensitizes cells to oxidative stress-induced apoptosis $(19,20)$, while the specific mechanism remains controversial. To investigate the relationship between HBx and MPTP in oxidative stress-induced apoptosis, we next examined the effects of an MPTP inhibitor for its ability to regulate the expression of proteins involved in the intrinsic apoptosis pathway in $\mathrm{H}_{2} \mathrm{O}_{2}$-exposed $\mathrm{HBx}$-expressing cells by western blot analysis. As shown in Fig. 5A and B, the expression of cleaved caspase- 3 and PARP was markedly decreased as a consequence of the release of cytosolic cytochrome $c$ in the $\mathrm{H}_{2} \mathrm{O}_{2}$-exposed $\mathrm{HBx}$-expressing cells. We also found a significant increase in the apoptosis level in the $\mathrm{H}_{2} \mathrm{O}_{2}$-exposed HL-7702-HBx cells by flow cytometric analysis (Fig. 5C and D), which was consistent with the immunoblot results. Additionally, MPTP inhibitor CsA apparently reduced the expression of cleaved caspase-3 and PARP and blocked the release of cytochrome $c$, ultimately preventing the extent of apoptosis.

\section{Discussion}

HBx is known to modulate numerous cellular pathways, including cell cycle regulation, activation of transcription factors, viral replication, ROS generation and regulation of apoptosis. Among all these activities, HBx-induced apoptosis is thought to directly impact the development of HBV-associated liver damage (3). Given the discrepancy of 

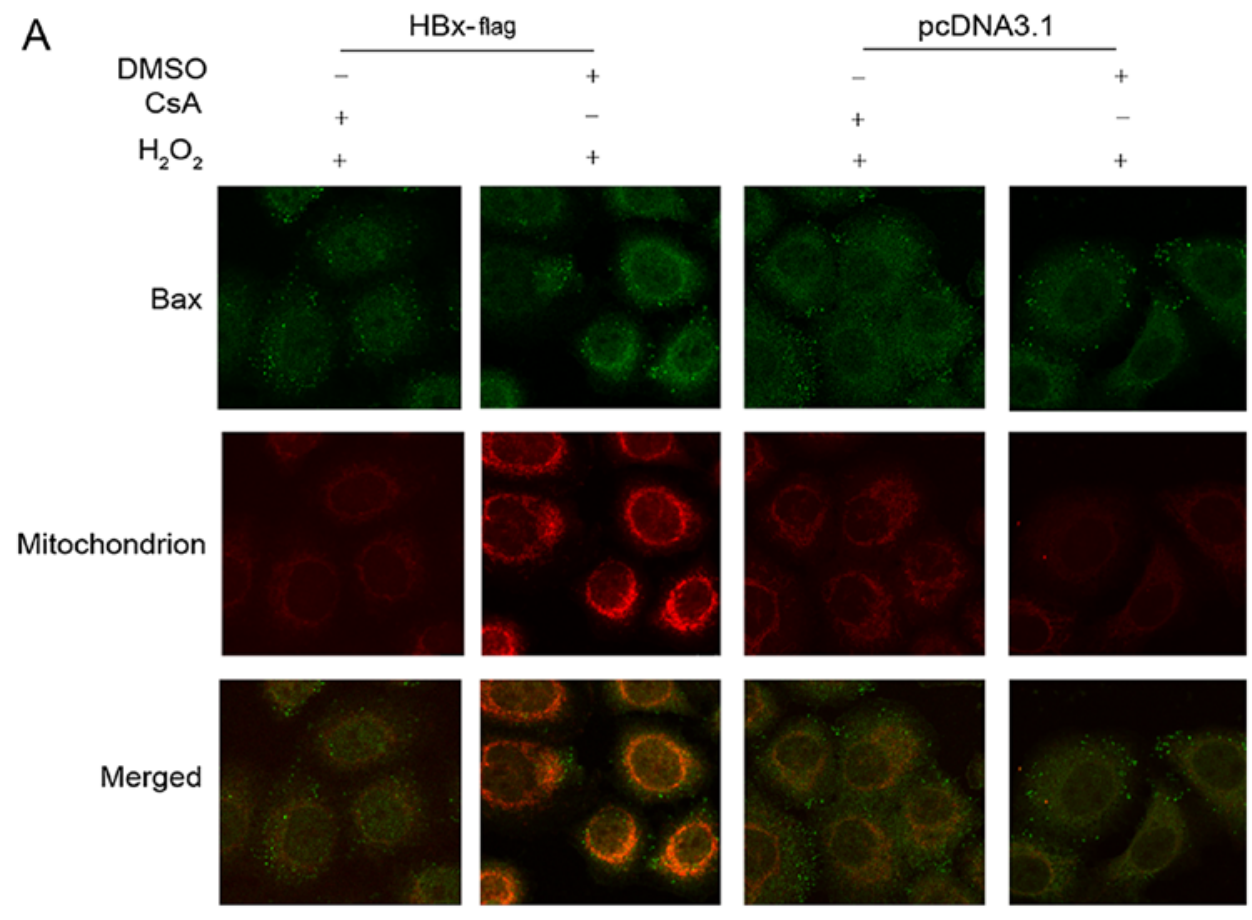

$\mathrm{B}$
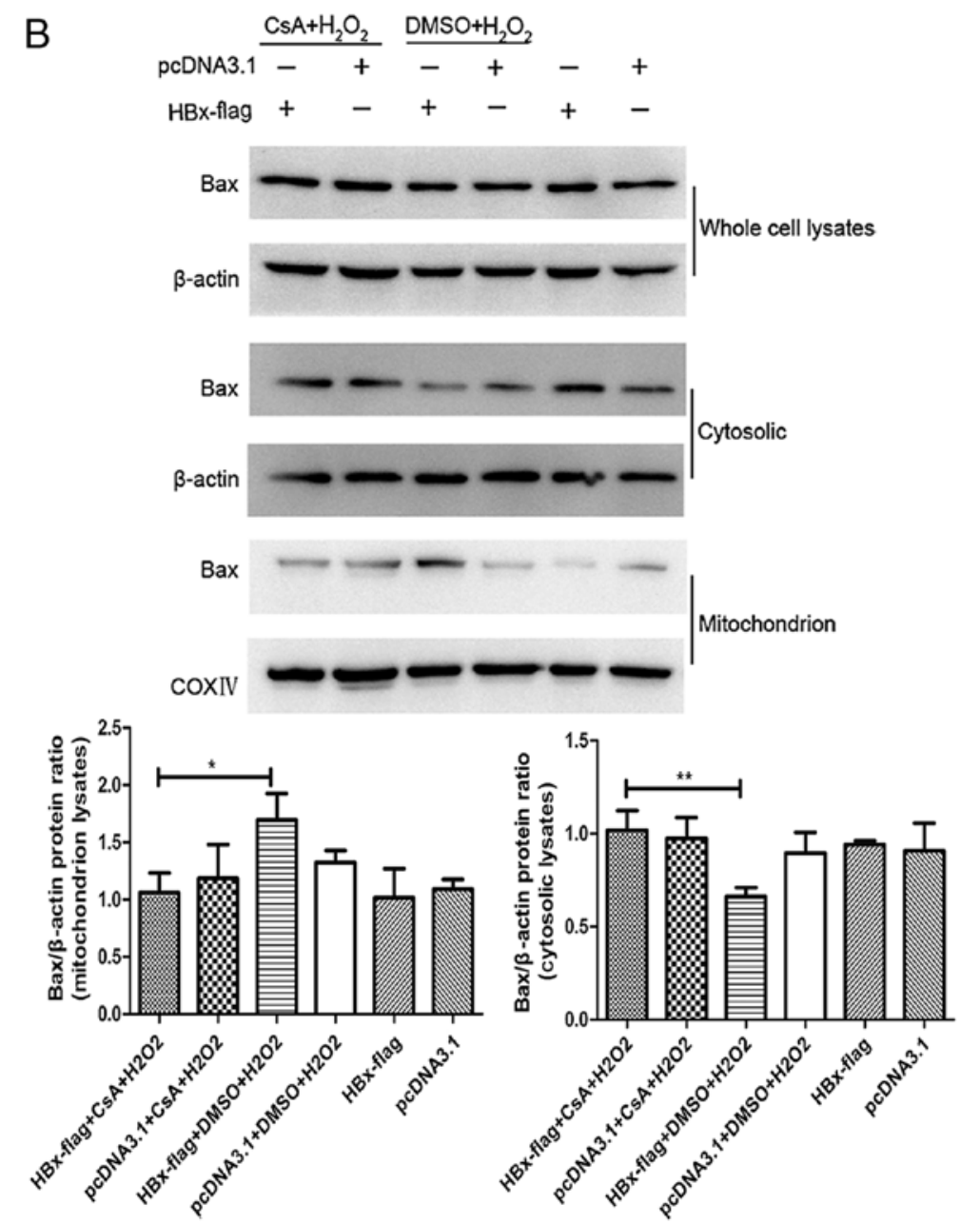

Figure 4. $\mathrm{HBx}$ induces translocation of Bax to mitochondria upon exposure to $\mathrm{H}_{2} \mathrm{O}_{2}$ by modulating MPTP. (A) Subcellular localizations of Bax and mitochondria were visualized by immunofluorescence staining with anti-Bax antibody (green) and MitoTracker (red). Magnification, x2,000. (B) The expression of Bax from whole cell cytosolic and mitochondrion lysates were analyzed by western blotting. Representative results are shown. ${ }^{*} \mathrm{P}<0.05$ and ${ }^{* * *} \mathrm{P}<0.01$.

the molecular mechanism of HBx in different cells, we usually conduct our research from the perspectives of a hepatic cell line and hepatoma cell line, respectively. In the present study, we explored the molecular mechanism responsible for 
A

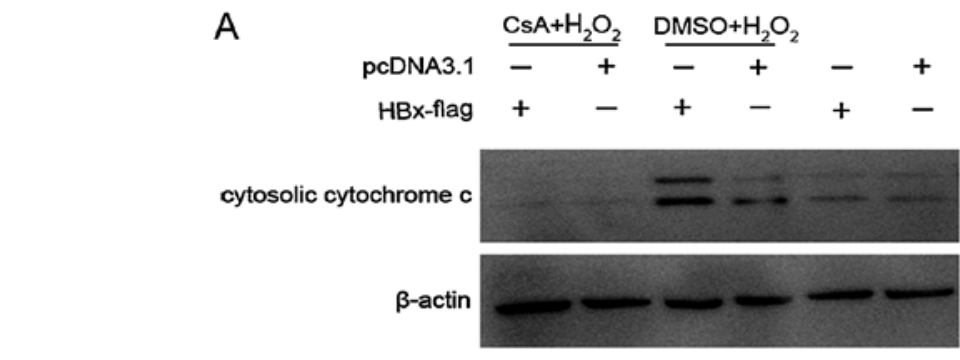

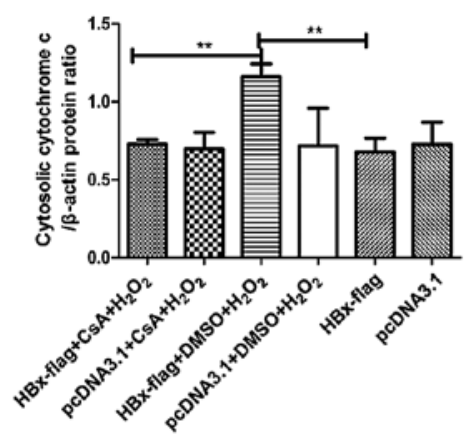
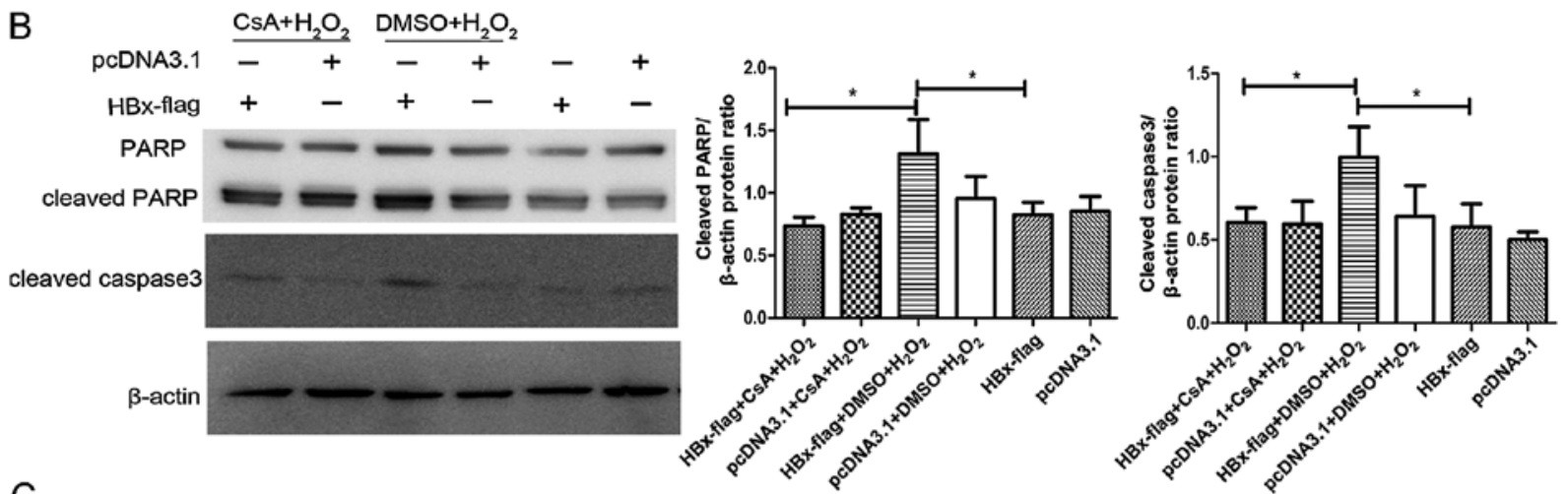

C
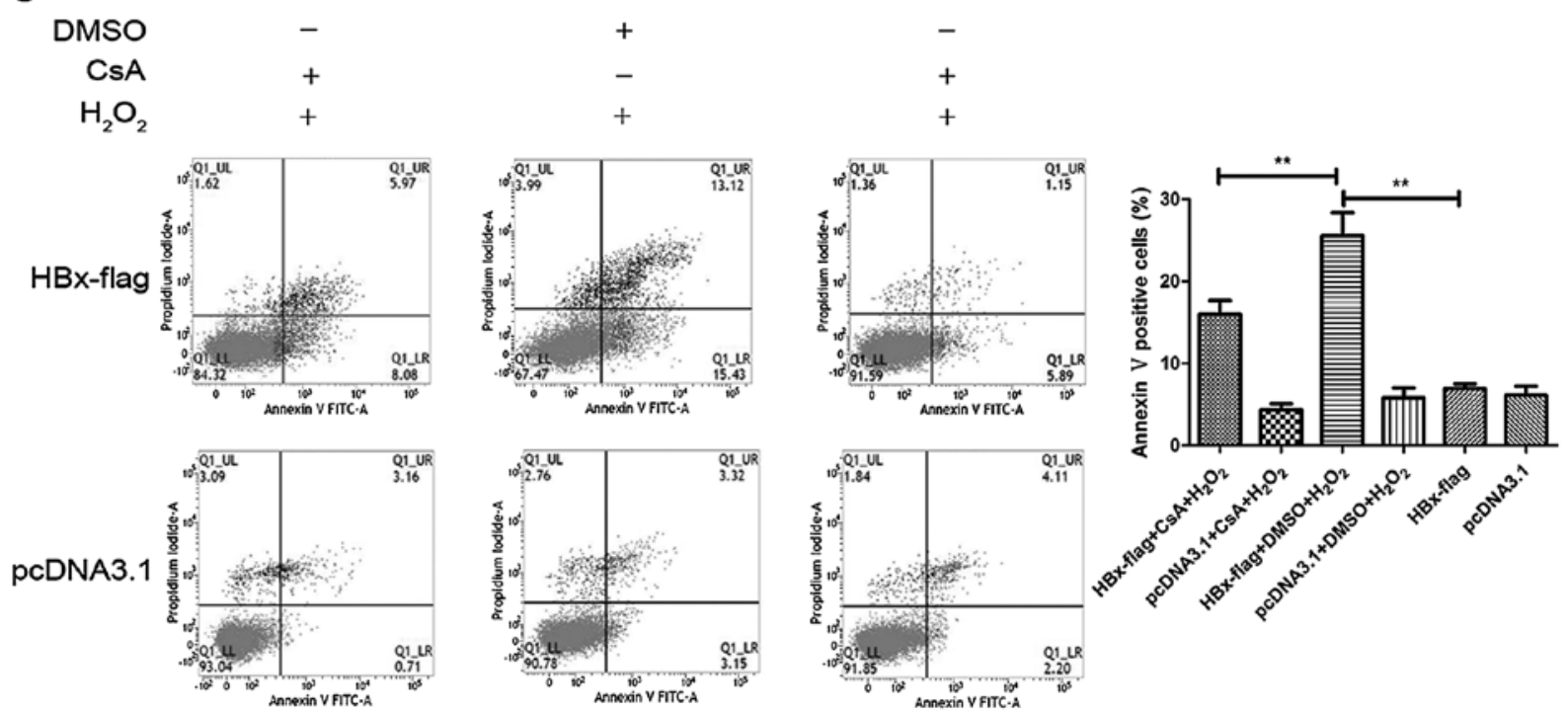

Figure 5. MPTP blockage with CsA attenuates the pro-apoptotic effect of HBx in response to oxidative stress. (A and B) Cells transfected with HBx-flag and pcDNA3.1 were treated with or without CsA followed by $\mathrm{H}_{2} \mathrm{O}_{2}$ exposure for $12 \mathrm{~h}$, and expression levels of cytosolic cytochrome $c$, PARP, cleaved caspase-3 and $\beta$-actin were determined by western blot assay. (C)The extent of apoptosis was evaluated by flow cytometric analysis. Values shown represent the mean \pm SD of three independent experiments; ${ }^{*} \mathrm{P}<0.05$ and ${ }^{* *} \mathrm{P}<0.01$

HBX-induced apoptosis upon exposure to oxidative stress in HL-7702, a human normal liver cell line.

MPTP is a large multi-protein complex and composed of voltage-dependent anion channel (VDAC), adenine nucleotide translocator (ANT), creatine kinase, hexokinase, the benzodiazapine receptor and cyclophilin D (27). It can form a large channel between cytosolic and mitochondrial matrix. Opening of MPTP in response to numerous mitochondrial stimuli is correlated with the swelling of mitochondria matrix with a release in molecules from the mitochondria matrix, including cytochrome $c$ and calcium, indicating that opening of MPTP may be an early event in apoptosis $(12,33)$. Various studies have reported that a fraction of cytosolic HBx co-localizes with the mitochondria in different cell lines, with studies in HepG2 cells suggesting that HBx could modulate the onset of MPTP to increase the level of cytosolic calcium, which contributes to the elevation of $\mathrm{HBV}$ replication, and this activity of $\mathrm{HBx}$ is blocked by treatment with CsA (11), a MPTP inhibitor. This result was consistent with the present study in HL-7702 cells that $\mathrm{HBx}$ has the ability to modulate MPTP, therefore causing a pronounced increase in cytosolic calcium levels. Additionally, another study reported that $\mathrm{HBx}$ requires MPTP activity to modulate the levels of cell cycle regulatory proteins (34). All these studies suggest that the opening of MPTP may contribute to the disruption of hepatocyte physiology. Involvement of the MPTP in HBx may be related to the co-localization 
of HBx and mitochondria, while the exact mechanism has not been fully understood. We previously reported a new HBx-interactive protein COXIII $(8,9,35,36)$, which is one of the subunits of cytochrome $c$ oxidase (COX) residing in the mitochondrial inner membrane in addition to VDAC3. COX functions as a terminal enzyme of the respiratory chain, and significantly influences the generation of ATP or depolarization of the mitochondrial membrane potential (37). The present study demonstrated that HBx significantly decreased the activity of COX, followed by a consistent reduction in cell ATP levels and mitochondrial membrane potential, both of which are responsible for the opening of the MPTP (38). As we predicted, cytosolic calcium overload was observed in the HBx-expressing HL-7702 cells as a consequence of MPTP opening, while the calcium levels were significantly reduced using CsA. The study in pHBV- and pHBV-HBx-expressing cells further confirmed these results. Thereby, it is possible that reduction in $\mathrm{COX}$ activity could be an initial factor in modulating the opening of the MPTP.

Persistent oxidative stress has been suggested to play a pivotal role in a wide variety of pathophysiological conditions $(13,21)$. Previous study in our laboratory showed that HBx expression could enhance ROS production $(8,9)$ compared with the control group. Other studies suggest that $\mathrm{HBx}$ induces apoptosis by sensitizing cells and tissues to $\mathrm{H}_{2} \mathrm{O}_{2}$ (19). Notably, ROS accumulation has been shown to upregulate $\mathrm{HBx}$ expression (39). In the present study, the concentration of $\mathrm{H}_{2} \mathrm{O}_{2}$ we used did not show an apparent change in ROS levels in $\mathrm{HBx}$-expressing cells or the control group, however, it induced a significant enhancement of ROS levels in the pHBV-expressing cells compared with the control group, demonstrating that $\mathrm{HBx}$-induced ROS accumulation in normal liver cells was dependent on constitutively replicated $\mathrm{HBV}$. When exposed to $\mathrm{H}_{2} \mathrm{O}_{2}$, a pronounced accumulation of ROS was observed in the HBx- and pHBVexpressing cells vs. the control groups, suggesting that $\mathrm{HBx}$ protein sensitized HL-7702 cells to oxidative stress. Results from previous studies showed that exposure to $\mathrm{H}_{2} \mathrm{O}_{2}$ caused the opening of MPTP, resulting in mitochondrial membrane depolarization and the release of cytochrome $c$ from mitochondria to the cytosol (12), while CsA prevents $\mathrm{H}_{2} \mathrm{O}_{2}$-induced apoptosis (40). Accordingly, modulation of MPTP by HBx may be a potential mechanistic link between $\mathrm{HBx}$ and oxidative stress-induced apoptosis.

Molecules of apoptosis can be released from the mitochondria through disruption in the outer mitochondrial membrane, as a consequence of the co-localization of $\mathrm{HBx}$ and mitochondria, pointing out an important role of $\mathrm{BcL}-2$ family protein which is made up of outer mitochondrial membrane proteins in the mitochondrial-mediator apoptosis pathway (41). Both of these proteins play a crucial role in maintaining mitochondrial membrane integrity (42). Various data demonstrated that pro-apoptotic Bax plays an important role in $\mathrm{H}_{2} \mathrm{O}_{2}$-induced apoptosis via translocation from cytosol to mitochondria with a release of cytochrome $c$ (25). In addition, VDAC, which is a component of MPTP, is required for the efficient translocation of Bax. During apoptotic stimuli, Bax is dissociated from VDAC2 and homoligomerizes to form high molecular weight oligomers (26), indicating that Bax translocation is probably dependent on the status of MPTP. In the present study, we found a significant co-localization of Bax and mitochondria in the HBx-expressing HL-7702 cells with a release of cytosolic cytochrome $c$ and activation of cleaved caspase-3 or PARP when exposed to $\mathrm{H}_{2} \mathrm{O}_{2}$. In $\mathrm{HBx}$-expressing cells pre-treated with CsA, this translocation of Bax was blocked. Given these observations, we propose that mitochondrial localization of Bax has a role in $\mathrm{HBx}$-induced apoptosis during oxidative stress. Moreover, this mechanism is associated with the function of $\mathrm{HBx}$ in modulating MPTP. Flow cytometric analysis further confirmed that an intracellular increase in $\mathrm{H}_{2} \mathrm{O}_{2}$ had a critical effect on HBx-induced apoptosis in HL-7702 cells, and this effect was abrogated by inhibition of MPTP. Our result is consistent with previous observations that $\mathrm{HBx}$ increases the susceptibility of different hepatocytes to apoptotic stimuli. Nevertheless, evidence supports an opposite role of $\mathrm{HBx}$, indicating a critical role of $\mathrm{HBx}$ in anti-apoptosis signals. The discrepant consequences of HBx expression on modulation of apoptosis may result from different cell lines and different stages of natural HBV infection. Accordingly, more research using hepatoma cell lines are needed in the future. $\mathrm{HBx}$-induced apoptosis during $\mathrm{H}_{2} \mathrm{O}_{2}$ exposure was significantly reduced with the pre-treatment of $\mathrm{CsA}$, indicating that modulating of MPTP may contribute to oxidative stressinduced apoptosis in HBx-expressing cells.

In summary, the present study in HL-7702 cells demonstrated that HBx enhances the susceptibility of normal hepatocytes to oxidative stress-induced apoptosis and this effect is associated with the ability of HBx to modulate MPTP, which is considered as the major cause of Bax mitochondrial translocation. This effect of HBx in response to oxidative stress may offer a new clue that HBx and oxidative signals may work together to upregulate cellular ROS to a deleterious level, thereby contributing to HBV-associated inflammation and hepatocarcinogenesis.

\section{Acknowledgements}

The present study was supported by the National Natural Science Foundation of China (81300321), the Key Clinical Specialty Discipline Construction Program of Fujian, China (Min Wei Ke Jiao 2012 No. 49), the Young and Middle-Aged Personnel Training Project of Fujian Province Health Department (2014-ZQN-ZD-9), and the Natural Science Foundation of Fujian Province, China (2016J05189). We thank Professor M.J. Bouchard for the $\mathrm{pHBV}$ and $\mathrm{pHBV}-\mathrm{HBx}$ plasmid.

\section{References}

1. Feitelson MA and Lee J: Hepatitis B virus integration, fragile sites, and hepatocarcinogenesis. Cancer Lett 252: 157-170, 2007.

2. Murakami S: Hepatitis B virus X protein: A multifunctional viral regulator. J Gastroenterol 36: 651-660, 2001.

3. Rawat S, Clippinger AJ and Bouchard MJ: Modulation of apoptotic signaling by the hepatitis $B$ virus $X$ protein. Viruses 4 : 2945-2972, 2012

4. Ma J, Sun T, Park S, Shen G and Liu J: The role of hepatitis B virus $X$ protein is related to its differential intracellular localization. Acta Biochim Biophys Sin 43: 583-588, 2011.

5. Huh KW and Siddiqui A: Characterization of the mitochondrial association of hepatitis B virus X protein, $\mathrm{HBx}$. Mitochondrion 1: 349-359, 2002.

6. Rahmani Z, Huh KW, Lasher R and Siddiqui A: Hepatitis B virus $X$ protein colocalizes to mitochondria with a human voltage-dependent anion channel, HVDAC3, and alters its transmembrane potential. J Virol 74: 2840-2846, 2000. 
7. Shirakata $\mathrm{Y}$ and Koike $\mathrm{K}$ : Hepatitis $\mathrm{B}$ virus $\mathrm{X}$ protein induces cell death by causing loss of mitochondrial membrane potential. J Biol Chem 278: 22071-22078, 2003.

8. Zou LY, Zheng BY, Fang XF, Li D, Huang YH, Chen ZX, Zhou LY and Wang XZ: HBx co-localizes with COXIII in HL-7702 cells to upregulate mitochondrial function and ROS generation. Oncol Rep 33: 2461-2467, 2015.

9. Zheng BY, Fang XF, Zou LY, Huang YH, Chen ZX, Li D Zhou LY, Chen $\mathrm{H}$ and Wang XZ: The co-localization of HBx and COXIII upregulates COX-2 promoting HepG2 cell growth. Int J Oncol 45: 1143-1150, 2014.

10. Gearhart TL and Bouchard MJ: Replication of the hepatitis B virus requires a calcium-dependent $\mathrm{HBx}$-induced $\mathrm{G} 1$ phase arrest of hepatocytes. Virology 407: 14-25, 2010.

11. McClain SL, Clippinger AJ, Lizzano R and Bouchard MJ: Hepatitis $\mathrm{B}$ virus replication is associated with an $\mathrm{HBx}$-dependent mitochondrion-regulated increase in cytosolic calcium levels. J Virol 81: 12061-12065, 2007.

12. Takeyama N, Miki S, Hirakawa A and Tanaka T: Role of the mitochondrial permeability transition and cytochrome $c$ release in hydrogen peroxide-induced apoptosis. Exp Cell Res 274: $16-24,2002$

13. Jaeschke H: Reactive oxygen and mechanisms of inflammatory liver injury: Present concepts. J Gastroenterol Hepatol 26 (Suppl 1): S173-S179, 2011.

14. Liang X, Liu Y, Zhang Q, Gao L, Han L, Ma C, Zhang L, Chen YH and Sun W: Hepatitis B virus sensitizes hepatocytes to TRAIL-induced apoptosis through Bax. J Immunol 178: 503-510, 2007.

15. Miao J, Chen GG, Chun SY and Lai PPS: Hepatitis B virus X protein induces apoptosis in hepatoma cells through inhibiting Bcl-xL expression. Cancer Lett 236: 115-124, 2006.

16. Rawat $\mathrm{S}$ and Bouchard MJ: The hepatitis B virus (HBV) $\mathrm{HBx}$ protein activates AKT to simultaneously regulate HBV replication and hepatocyte survival. J Virol 89: 999-1012, 2015.

17. Shen L, Zhang X, Hu D, Feng T, Li H, Lu Y and Huang J: Hepatitis B virus $X(\mathrm{HBx})$ play an anti-apoptosis role in hepatic progenitor cells by activating Wnt/ $\beta$-catenin pathway. Mol Cell Biochem 383: 213-222, 2013

18. Madden CRF, Finegold MJ and Slagle BL: Expression of hepatitis $\mathrm{B}$ virus $\mathrm{X}$ protein does not alter the accumulation of spontaneous mutations in transgenic mice. J Virol 74: 5266-5272, 2000.

19. Hu L, Chen L, Yang G, Li L, Sun H, Chang Y, Tu Q, Wu M and Wang H: HBx sensitizes cells to oxidative stress-induced apoptosis by accelerating the loss of Mcl-1 protein via caspase-3 cascade. Mol Cancer 10: 43, 2011.

20. Kim WH, Hong F, Jaruga B, Zhang ZS, Fan SJ, Liang TJ and Gao B: Hepatitis B virus X protein sensitizes primary mouse hepatocytes to ethanol- and TNF-alpha-induced apoptosis by a caspase-3-dependent mechanism. Cell Mol Immunol 2: 40-48, 2005.

21. Brady NR, Hamacher-Brady A, Westerhoff HV and Gottlieb RA: A wave of reactive oxygen species (ROS)-induced ROS release in a sea of excitable mitochondria. Antioxid Redox Signal 8: 1651-1665, 2006

22. Breckenridge DG and Xue D: Regulation of mitochondrial membrane permeabilization by BCL-2 family proteins and caspases. Curr Opin Cell Biol 16: 647-652, 2004

23. Westphal D, Dewson G, Czabotar PE and Kluck RM: Molecular biology of Bax and Bak activation and action. Biochim Biophys Acta 1813: 521-531, 2011.

24. Kim HJ, Kim SY, Kim J, Lee H, Choi M, Kim JK and Ahn JK: Hepatitis B virus $\mathrm{X}$ protein induces apoptosis by enhancing translocation of Bax to mitochondria. IUBMB Life 60: 473-480 2008.

25. Ahmad KAIK, Iskandar KB, Hirpara JL, Clement MV and Pervaiz S: Hydrogen peroxide-mediated cytosolic acidification is a signal for mitochondrial translocation of Bax during drug-induced apoptosis of tumor cells. Cancer Res 64: 7867-7878, 2004 .
26. Ma SB, Nguyen TN, Tan I, Ninnis R, Iyer S, Stroud DA, Menard M, Kluck RM, Ryan MT and Dewson G: Bax targets mitochondria by distinct mechanisms before or during apoptotic cell death: A requirement for VDAC2 or Bak for efficient Bax apoptotic function. Cell Death Differ 21: 1925-1935, 2014.

27. Lemasters JJ, Nieminen AL, Qian T, Trost LC, Elmore SP, Nishimura Y, Crowe RA, Cascio WE, Bradham CA, Brenner DA, et al: The mitochondrial permeability transition in cell death: A common mechanism in necrosis, apoptosis and autophagy. Biochim Biophys Acta 1366: 177-196, 1998.

28. Melegari M, Scaglioni P-P and Wands JR: Cloning and characterization of a novel hepatitis $\mathrm{B}$ virus $\mathrm{x}$ binding protein that inhibits viral replication. J Virol 72: 1737-1743, 1998.

29. Scaglioni PP, Melegari M and Wands JR: Posttranscriptional regulation of hepatitis $B$ virus replication by the precore protein. J Virol 71: 345-353, 1997.

30. Siletsky SA and Konstantinov AA: Cytochrome $c$ oxidase: Charge translocation coupled to single-electron partial steps of the catalytic cycle. Biochim Biophys Acta 1817: 476-488, 2012.

31. Li H, Wang B, Zhu C, Feng Y, Wang S, Shahzad M, Hu C, Mo M Du F and Yu X: 17 $\beta$-estradiol impedes Bax-involved mitochondrial apoptosis of retinal nerve cells induced by oxidative damage via the phosphatidylinositol 3-kinase/Akt signal pathway. J Mol Neurosci 50: 482-493, 2013.

32. Schellenberg B, Wang P, Keeble JA, Rodriguez-Enriquez R, Walker S, Owens TW, Foster F, Tanianis-Hughes J, Brennan K, Streuli $\mathrm{CH}$, et al: Bax exists in a dynamic equilibrium between the cytosol and mitochondria to control apoptotic priming. Mol Cell 49: 959-971, 2013.

33. Yang JC and Cortopassi GA: Induction of the mitochondrial permeability transition causes release of the apoptogenic factor cytochrome $c$. Free Radic Biol Med 24: 624-631, 1998.

34. Clippinger AJ, Gearhart TL and Bouchard MJ: Hepatitis B virus $\mathrm{X}$ protein modulates apoptosis in primary rat hepatocytes by regulating both NF-kappaB and the mitochondrial permeability transition pore. J Virol 83: 4718-4731, 2009.

35. Li D, Wang XZ, Yu JP, Chen ZX, Huang YH and Tao QM: Cytochrome $c$ oxidase III interacts with hepatitis B virus $X$ protein in vivo by yeast two-hybrid system. World J Gastroenterol 10: 2805-2808, 2004.

36. Wang XZ, Li D, Tao QM, Lin $\mathrm{N}$ and Chen ZX: A novel hepatitis B virus X-interactive protein: Cytochrome $c$ oxidase III. J Gastroenterol Hepatol 21: 711-715, 2006.

37. Mkaouar-Rebai E, Ellouze E, Chamkha I, Kammoun F, Triki C and Fakhfakh F: Molecular-clinical correlation in a family with a novel heteroplasmic Leigh syndrome missense mutation in the mitochondrial cytochrome $c$ oxidase III gene. J Child Neurol 26: 12-20, 2011.

38. Tsujimoto $Y$, Nakagawa $\mathrm{T}$ and Shimizu S: Mitochondrial membrane permeability transition and cell death. Biochim Biophys Acta 1297-1300: 2006.

39. Wang JH, Yun C, Kim S, Lee JH, Yoon G, Lee MO and Cho H: Reactive oxygen species modulates the intracellular level of $\mathrm{HBx}$ viral oncoprotein. Biochem Biophys Res Commun 310: 32-39, 2003.

40. Sugano N, Ito $\mathrm{K}$ and Murai S: Cyclosporin A inhibits $\mathrm{H}_{2} \mathrm{O}_{2}$-induced apoptosis of human fibroblasts. FEBS Lett 447 : 274-276, 1999.

41. Czabotar PE, Lessene G, Strasser A and Adams JM: Control of apoptosis by the BCL-2 protein family: Implications for physiology and therapy. Nat Rev Mol Cell Biol 15: 49-63, 2014.

42. Harris $\mathrm{MH}$ and Thompson CB: The role of the Bcl-2 family in the regulation of outer mitochondrial membrane permeability. Cell Death Differ 7: 1182-1191, 2000. 